\title{
L'enseignement technologique et professionnel français... et la formation tout au long de la vie
}

Benoît Bouyx

\section{OpenEdition}

1 Journals

Édition électronique

URL : http://journals.openedition.org/ries/3042

DOI : 10.4000/ries.3042

ISSN : 2261-4265

Éditeur

Centre international d'études pédagogiques

Édition imprimée

Date de publication : 1 décembre 1997

Pagination : 89-100

ISSN : 1254-4590

\section{Référence électronique}

Benoît Bouyx, « L'enseignement technologique et professionnel français... et la formation tout au long de la vie », Revue internationale d'éducation de Sèvres [En ligne], 16 | Décembre 1997, mis en ligne le 07 juin 2013, consulté le 19 avril 2019. URL : http://journals.openedition.org/ries/3042 ; DOI : 10.4000/ ries.3042

Ce document a été généré automatiquement le 19 avril 2019

(c) Tous droits réservés 


\title{
L'enseignement technologique et professionnel français... et la formation tout au long de la vie
}

\author{
Benoît Bouyx
}

\section{NOTE DE L'AUTEUR}

Voir également pour un développement plus complet, Benoît Bouyx, L'enseignement technologique et professionnel, Paris, CNDP/Documentation française, janvier 1997.

1 Les lois de 1971, appelées souvent lois Delors, ont institué en France la formation continue dans le cadre de l'éducation permanente, expression au sens multiforme renvoyant aussi bien à l'adaptation des travailleurs aux évolutions technologiques et organisationnelles (mais ce n'était pas l'axe majeur développé à l'époque!) qu'à l'égalisation des chances et au droit à la deuxième chance et, surtout, à l'accès des travailleurs à la culture ${ }^{1}$.

2 Le bilan qu'on peut dresser de la mise en œuvre de ces lois trente cinq ans après, apparaît bien dans un numéro récent d'Éducation permanente intitulé « Formation des adultes: de l'utopie à la Crise $»^{2}$ avec des articles au titre hautement significatif: "La formation continue accroît-elle aujourd'hui les inégalités?» de Claude Dubar qui apporte une réponse personnelle positive, ou «L'érosion des idéaux de l'éducation permanente » de Jean-Pierre Le Goff. Il est vrai que le contexte économique et social a radicalement changé et que les lois de 1971 portent la marque de la fin des trente glorieuses et du plein emploi, tandis que la situation actuelle est dominée par le chômage et la menace qu'il fait peser sur chacun d'entre nous. Il est difficile pour ceux qui ont œuvré au développement de la formation continue en France depuis trente ans, de partager l'enthousiasme des auteurs du Livre blanc de la Communauté européenne, Enseigner et apprendre - Vers la société 
cognitive, et de la formule qui l'accompagne d'éducation et formation tout au long de la vie.

3 C'est ainsi que l'année 1996 a été déclarée Année européenne de l'éducation et de la formation tout au long de la vie et que cette expression fait maintenant partie de la toile de fond de tous les débats sur la formation. Si on ne peut qu'adhérer à l'idée que l' éducation et la formation concernent toutes les étapes de la vie et reconnaître que, jusqu' à un passé récent - et encore bien souvent aujourd'hui - on ne pense que formation initiale, il paraît difficile de réfléchir à la nécessaire articulation entre formation initiale et formation continue sans analyser les raisons qui ont conduit à l'émergence de cette formule et à son adoption rapide dans tous les États de la Communauté européenne, au détriment du concept d'éducation permanente devenu presque désuet.

Ces raisons apparaissent fort bien dans le Livre blanc de la Commission européenne paru en 1995 Enseigner et apprendre - Vers la société cognitive. Il pose comme postulat que trois facteurs de transformation de la société sont incontournables et s'imposent à l'Europe : l'entrée dans la société de l'information, la mondialisation des échanges, l'entrée dans une civilisation scientifique et technique. Si ces évolutions sont incontestables, on peut cependant s'interroger sur leur amplitude : introduisent-elles une rupture si radicale ? et sur les conséquences qui lui sont liées, notamment sur l'apparition d'un marché du travail flexible renversant de manière inéluctable la tendance passée au développement du travail salarié permanent. La conséquence ultime en est la mise en cause du diplôme qui rigidifie le marché du travail et éliminerait les talents ne correspondant pas aux standards.

5 Ce n'est évidemment pas dans cette optique que le système français de formation professionnelle initiale et continue évolue et essaie de prendre en compte la nécessité de se former tout au long de la vie. Un système efficace d'éducation permanente n'a de chance de fonctionner que s'il est articulé avec un système de formation initiale performant pour l'ensemble des classes d'âge. Et c'est bien là le problème majeur de tout système éducatif que sa capacité à offrir une scolarisation de qualité pour $100 \%$ des jeunes.

6 Or, dans pratiquement tous les pays de l'Union, on s'aperçoit que l'enseignement professionnel initial joue un rôle très important dans la réussite ou non d'une scolarisation de masse. Au regard des statistiques européennes, les pourcentages d'élèves dans le deuxième cycle de l'enseignement secondaire sont en France de $55 \%$ pour l'enseignement professionnel et de $45 \%$ pour l'enseignement général.

7 Cet enseignement a connu ces dernières années des évolutions très importantes que cet article se propose de présenter à travers trois des principales mesures prises: la suppression du palier d'orientation vers les formations professionnelles après la septième année de scolarisation et son report après la neuvième année, la réorganisation des formations professionnelles de base en trente-quatre spécialités, la création et le développement d'une nouvelle catégorie de baccalauréat : le baccalauréat professionnel ${ }^{3}$.

\section{Formation professionnelle et échec scolaire}

8 Jusqu'en 1980, la première orientation massive vers les formations professionnelles se faisait après sept ans de scolarisation (scolarité primaire et classe de $6^{\mathrm{e}}$ et $5^{\mathrm{e}}$ ). Elle débouchait sur la préparation au certificat d'aptitude professionnelle (le CAP) en trois ans 
pour environ $13,5 \%$ des élèves. Deux phénomènes allaient imposer un changement : la dégradation de l'insertion professionnelle des titulaires de CAP, accentuée par les phénomènes de concurrence entre les différents publics à la recherche d'un emploi, d'une part; le discours de plus en plus fort sur l'évolution du contenu du travail, d'autre part. C'est l'époque où l'on commence à dire que plus personne ne fera le même métier tout au long de la vie, c'est aussi l'époque des grandes restructurations industrielles et des difficultés de reconversion de la main-d'œuvre vers d'autres types d'emplois.

Pour supprimer cette voie de formation professionnelle, tout en continuant à offrir une voie de scolarisation pour ces jeunes rejetés du collège traditionnel, de nouvelles formes de classes de $4^{\mathrm{e}}$ et $3^{\mathrm{e}}$ ont été mises au point, les classes de quatrième et troisième technologiques, dont l'objectif est de permettre à ces jeunes d'atteindre la fin du collège, en s'appuyant sur la technologie, mais sans préparer un métier.

10 On demande donc aujourd'hui au collège à la française de traiter l'ensemble des jeunes de chaque classe d'âge. On le demandait en fait depuis l'introduction de la scolarité obligatoire jusqu'à 16 ans (c'est-à-dire depuis 1959); mais on ne lui a pas demandé de modifier ses objectifs, ni posé le problème du modèle culturel qu'il diffuse. Le collège n'a jamais cessé d'être avant tout la structure qui prépare l'entrée dans un autre cursus généraliste : le lycée général. On attendait donc du collège, pensé pour une élite (10\% d'une classe d'âge accédait au baccalauréat à la fin des années cinquante) qu'il traite l'ensemble d'une classe d'âge avec les mêmes objectifs.

11 L'entrée dans une société où l'on se formera tout au long de la vie pose le problème des objectifs de la scolarité obligatoire et du consensus autour de ces objectifs. Le collège français reste dominé par l'acquisition de connaissances, séparées en disciplines autonomes fortement modelées à partir des savoirs universitaires et, comme ces objectifs ne peuvent pas être atteints par l'ensemble des jeunes, le remède miracle de l'orientation précoce vers les formations professionnelles resurgit en permanence.

12 Ce remède miracle est de plus en plus un leurre et on n'ira pas vers la formation tout au long de la vie si les bases ne sont pas assurées. Il convient donc de dissocier définitivement formation professionnelle initiale et remédiation à l'échec scolaire. Le problème du système éducatif est d'améliorer le fonctionnement du collège, de manière à ce qu'il traite mieux la totalité des élèves.

\section{La formation professionnelle de base : du CAP au BEP}

13 Après la fin du collège, environ un tiers d'une classe d'âge suit une scolarité préparant au brevet d'études professionnelles (BEP). Ce diplôme est devenu aujourd'hui le diplôme de base de la formation professionnelle, 275250 candidats s'y était présentés en 1996, contre 171151 au certificat d'aptitude professionnelle (si on élimine les candidats au BEP qui se présentent en même temps au CAP).

14 Le BEP est organisé en 34 spécialités, alors que le CAP l'est en 220, ce qui donne une idée du spectre visé par le diplôme davantage centré sur des familles professionnelles que sur des métiers. L'ensemble des contenus a été repensé de manière à reconstruire le moins mauvais compromis possible entre insertion professionnelle, capacité à la poursuite d'étude, capacité à la formation tout au long de la vie... poursuite d'études, la majorité des élèves de l'enseignement professionnel ayant 
désormais la possibilité de poursuivre après leur scolarité jusqu'au baccalauréat, grâce à la création d'une nouvelle catégorie de baccalauréat : le baccalauréat professionnel.

\section{La création du baccalauréat professionnel}

Le baccalauréat professionnel, créé en 1985 et auquel se sont présentés 90787 candidats en 1996 (soit environ $15 \%$ de l'ensemble des candidats au baccalauréat), est né de la convergence de trois facteurs :

- la volonté de mieux préparer les jeunes aux défis économiques de l'an 2000, les formations traditionnelles sanctionnées par un CAP ou un BEP semblant insuffisantes dans de nombreux secteurs en particulier dans tous ceux touchés par les nouvelles technologies de l'information ;

- l'analyse des niveaux de formation des sortants du système éducatif et le constat que très peu de jeunes sortaient du système éducatif au niveau du baccalauréat, le baccalauréat étant très majoritairement un tremplin pour poursuivre des études - a contrario de très nombreux jeunes entraient sur le marché du travail avec un niveau CAP-BEP (ce que l'on appelle, en France, le niveau $\mathrm{V}$ de formation) ou avec un niveau III (BTS, DUT);

- la volonté de décloisonner les lycées professionnels et les centres de formation d'apprentis, enfin. Ceux-ci avaient la réputation de n'offrir que des formations « cul de sac » avec comme perspective unique l'entrée sur le marché du travail ou... le chômage à l'issue de la formation ; la formation continue (ou la formation tout au long de la vie...) ne présentant pas une alternative crédible face aux voies d'enseignement général.

17 Ainsi fut décidé de créer un nouveau type de baccalauréat : le baccalauréat professionnel qui présente plusieurs originalités par rapport aux autres baccalauréats. Il n'est accessible qu'après un premier diplôme professionnel (essentiellement le BEP correspondant à deux années d'études après la classe de $3^{\mathrm{e}}$ ) et se prépare en deux années supplémentaires : il s'agit d'une formation en quatre ans après la $9^{\mathrm{e}}$ année. Ce choix a permis d'offrir une possibilité de poursuite d'études positive pour des élèves qui en étaient exclus auparavant.

Il comporte des périodes obligatoires de formation en entreprise d'une durée de douze à vingt-quatre semaines selon les spécialités, la durée moyenne étant de seize semaines, c'est-à-dire de quatre mois. Par ailleurs, les acquis de ces périodes sont pris en compte dans l'examen final. Il s'agit là d'une innovation assez fondamentale dans le système éducatif français, qui marque par certains côtés la fin de l'opposition traditionnelle entre l'école et l'entreprise. Ce choix correspond à une prise de conscience du fait que certaines compétences ne peuvent s'acquérir qu'en situation réelle ou en vraie grandeur : c'est par exemple particulièrement vrai pour les formations à la maintenance dans le secteur industriel ou à la vente dans le secteur des services. Jusqu'alors, le lycée professionnel était traditionnellement une usine en modèle réduit mais ce qui était possible dans un monde peu évolutif ne l'est plus aujourd'hui. L'apparition des technologies nouvelles se traduit par une complexité plus grande des processus de production et une plus grande autonomie des opérateurs. Ils doivent ainsi de plus en plus réagir aux aléas et seul l'exercice de l'activité professionnelle en situation réelle peut permettre d'acquérir les compétences correspondantes. C'est une évolution profonde pour les entreprises françaises dont on reconnaît la fonction "formatrice ", ce qui ne correspond guère à la tradition française, et pour le système éducatif appelé à reconnaître qu'une formation professionnelle complète passe nécessairement par l'entreprise. 
19 L'examen final est complètement repensé. Pour la première fois dans un diplôme français, les acquis des périodes de formation en entreprise sont évalués à l'examen et le système traditionnel d'épreuves identiques pour tous les candidats est remplacé par un système mixte reposant pour certaines épreuves sur le maintien d'un contrôle ponctuel terminal et, pour d'autres, par un système de contrôle en cours de formation confié aux équipes pédagogiques. Cette formule présente de nombreux avantages : elle supprime une partie du caractère aléatoire de l'examen, elle permet une meilleure adaptation de la formation aux situations réelles et à l'environnement économique local.

Le baccalauréat professionnel a connu un développement très rapide puisque plus de 90000 candidats s'y sont présentés en 1996 alors que sa première session avait eu lieu en 1987. Il contribue ainsi très fortement à l'augmentation des jeunes qui obtiennent le baccalauréat : 62,7\% de la classe d'âge en 1995 au lieu de $30 \%$ dix ans auparavant, la part du baccalauréat professionnel étant de $13,5 \%$.

21 Ce nouveau diplôme pose de manière incontournable le problème de la liaison entre formation initiale et formation continue et donc le problème de la formation tout au long de la vie. Il a le titre de baccalauréat et, en France, le baccalauréat est le premier grade de l'enseignement supérieur. Dans son intitulé, il porte en germe la tentation de la poursuite d'études initiales. Cela ne correspond pourtant pas à la volonté de ceux qui ont choisi ce titre en pensant que compte tenu de la place du baccalauréat en France, il fallait si l'on voulait revaloriser la voie professionnelle donner aussi à ce nouveau diplôme le titre de baccalauréat - mais un baccalauréat visant d'abord l'insertion professionnelle.

Les titulaires du baccalauréat professionnel ont vocation à s'insérer majoritairement dans les emplois d'ouvriers et d'employés, mais ils y arrivent avec un potentiel et des attentes qui posent le problème de leur carrière. La vraie solution, et ce serait sans doute une grande innovation en France qui nous rapprocherait du modèle allemand, résiderait dans une meilleure articulation entre formation initiale et formation continue, c'est-à-dire dans la possibilité de se promouvoir tout au long de la vie professionnelle.

Cet article s'est volontairement limité à la formation des ouvriers et des employés, car c'est là que résident les véritables enjeux, en particulier, si l'on se soucie de traiter l'ensemble des jeunes de chaque classe d'âge. En présentant les évolutions actuelles du système éducatif français dans le domaine de la formation professionnelle initiale, je me suis efforcé de montrer que la formation tout au long de la vie ne sera qu'un slogan si la formation continue ne s'articule pas sur une formation initiale qui arme pour la suite de la vie et n'arme pas seulement les meilleurs, mais tous les jeunes.

24 La formation tout au long de la vie bien sûr, mais pour tous et donc pas seulement pour l'élite qui a déjà bénéficié de la meilleure formation initiale, comme en France où plus de vingt ans après la mise en place de la formation continue, on s'aperçoit que ceux qui en profitent le plus sont ceux qui ont la formation initiale la plus solide. L'enjeu est donc d'être capable de mettre $100 \%$ d'une classe d'âge en position de profiter vraiment de la formation tout au long de la vie. 


\section{NOTES}

1. Denis Kalien, «Éducation permanente une rétrospective », Formation professionnelle, $\mathrm{n}$ • 8/9, mai-décembre 1996, p. 16-22.

2. «Formation des adultes : de l'utopie à la crise ", Éducation permanente, n 129, mars 1997.

3. Notamment, Alain d'Iribarne, « Une lecture des paradigmes du Livre blanc sur l'éducation et la formation », Revue du CEDEFOP, n 8-9, 1996, p. 23-32.

\section{RÉSUMÉS}

Étudiant les évolutions de la formation initiale et professionnelle d'une catégorie de la population, celle des ouvriers et employés, l'auteur démontre, preuves à l'appui, qu'il y a nécessité à reformuler les objectifs de la formation initiale et à reconsidérer à la fois, l'orientation en fin de collège et le rôle des diplômes. À ses yeux, c'est la validation des acquis professionnels qui offre un espace de recomposition.

\section{INDEX}

Index géographique : France

Mots-clés : enseignement professionnel, enseignement technique, examen, formation continue

\section{AUTEUR}

\section{BENOÎT BOUYX}

Direction des lycées et des collèges, ministère de l'Éducation nationale, de la Recherche et de la Technologie, Paris, France 